\title{
ERRATUM
}

Erratum: Kinetic inductance detectors for the OLIMPO experiment: design and pre-flight characterization

To cite this article: A. Paiella et al JCAP02(2019)E01

View the article online for updates and enhancements.

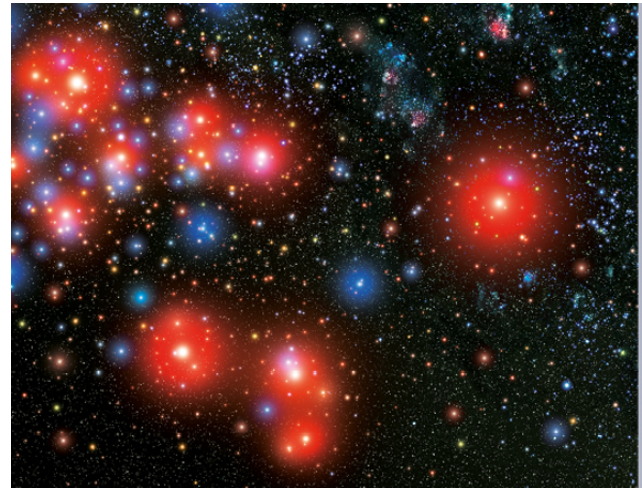

A.A IOP Astronomy ebooks

Part of your publishing universe and your first choice for

astronomy, astrophysics, solar physics and planetary

science ebooks

iopscience.org/books/aas 


\section{Erratum: Kinetic inductance detectors for the OLIMPO experiment: design and pre-flight characterization}

A. Paiella, ${ }^{a, b, 1}$ A. Coppolecchia, ${ }^{a, b}$ L. Lamagna, ${ }^{a, b}$ P.A.R. Ade, ${ }^{c}$ E.S. Battistelli, ${ }^{a, b}$ M.G. Castellano, ${ }^{d}$ I. Colantoni, ${ }^{d, 2}$ F. Columbro, ${ }^{a, b}$ G. D’Alessandro, ${ }^{a, b}$ P. de Bernardis, ${ }^{a, b}$ S. Gordon, ${ }^{e}$ S. Masi, ${ }^{a, b}$ P. Mauskopf,,$^{e, f}$ G. Pettinari, ${ }^{d}$ F. Piacentini, ${ }^{a, b}$ G. Pisano, ${ }^{c}$ G. Presta ${ }^{a, b}$ and C. Tucker ${ }^{c}$

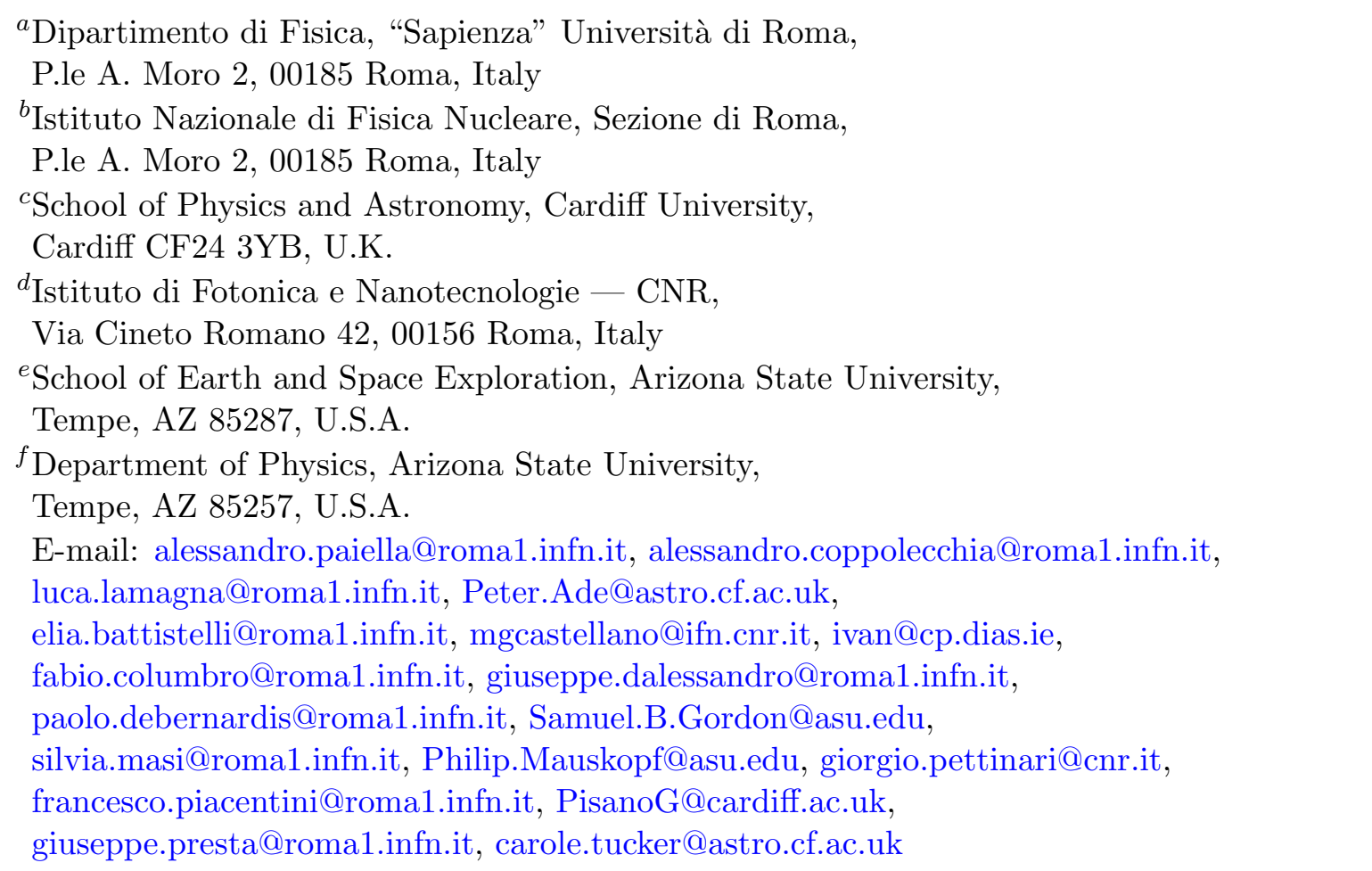

Received February 1, 2019

Accepted February 1, 2019

Published February 5, 2019

Erratum to: JCAP01(2019)039

ArXiv ePrint: 1810.00598

\footnotetext{
${ }^{1}$ Corresponding author.

${ }^{2}$ Current address: School of Cosmic Physics, Dublin Institute for Advanced Studies, 31 Fitzwilliam Place, D02 XF86, Dublin, Ireland.
} 


\section{Changes}

Table 1 would have to be changed with the following. There was a mistake in the evaluation of $\mathrm{NET}_{\mathrm{CMB}}$.

\begin{tabular}{|c|c|c|c|c|c|c|c|c|}
\hline OLIMPO configuration & \multicolumn{3}{|c|}{ Photometric } & \multicolumn{4}{c|}{ Spectrometric } \\
\hline Channel $[\mathrm{GHz}]$ & 150 & 250 & 350 & 460 & 150 & 250 & 350 & 460 \\
\hline Background Power $[\mathrm{pW}]$ & 2.7 & 14 & 4.9 & 13 & 7.6 & 50 & 15 & 33 \\
optical NEP $[\mathrm{aW} / \sqrt{\mathrm{Hz}}]$ & 65 & 140 & 90 & 140 & 110 & 260 & 160 & 240 \\
optical NET $_{\mathrm{CMB}}[\mu \mathrm{K} \sqrt{\mathrm{s}}]$ & 110 & 110 & 850 & 3300 & 190 & 550 & 1400 & 5200 \\
optical NET $_{\mathrm{RJ}}[\mu \mathrm{K} \sqrt{\mathrm{s}}]$ & 60 & 30 & 70 & 60 & 110 & 60 & 120 & 110 \\
\hline
\end{tabular}

Table 3 would have to be changed with the following. There was a mistake in the units of "Range".

\begin{tabular}{|c|c|c|c|c|c|c|c|}
\hline \multirow{3}{*}{$\begin{array}{l}\text { Channel } \\
{[\mathrm{GHz}]}\end{array}$} & \multirow{3}{*}{$\begin{array}{l}\# \text { of } \\
\text { pixels }\end{array}$} & \multicolumn{4}{|c|}{ Resonant frequencies } & \multirow{2}{*}{\multicolumn{2}{|c|}{$\begin{array}{l}\text { Maximum non-uniformity in the } \\
\text { inductor current distribution }\end{array}$}} \\
\hline & & \multirow{2}{*}{$\begin{array}{l}\text { Range } \\
{[\mathrm{MHz}]}\end{array}$} & \multirow{2}{*}{$\begin{array}{c}\text { Bandwidth } \\
{[\mathrm{MHz}]}\end{array}$} & \multicolumn{2}{|c|}{ Spacing $[\mathrm{MHz}]$} & & \\
\hline & & & & $1-2$ & Average & First pixel & Last pixel \\
\hline 150 & 23 & {$[120 ; 220]$} & 100 & 5 & 4.3 & $5 \%$ & $9 \%$ \\
\hline 250 & 39 & {$[125 ; 300]$} & 175 & 5 & 4.5 & $4 \%$ & $10 \%$ \\
\hline 350 & 25 & {$[305 ; 420]$} & 115 & 5 & 4.6 & $15 \%$ & $10 \%$ \\
\hline 460 & 43 & {$[230 ; 445]$} & 215 & 5 & 5 & $13 \%$ & $18 \%$ \\
\hline
\end{tabular}

These changes do not affect the results and the conclusion. 